\title{
Some peculiarities of modeling defects in polyaramide composite materials
}

\author{
by W. Świderski*, V. Vavilov** \\ * Military Institute of Armament Technology, Zielonka, Poland \\ ** Tomsk Polytechnic University, Tomsk, Russia
}

\begin{abstract}
Thin plane structures made of polyaramide composite have been studied experimentally and theoretically. By comparing experimental and theoretical results, it has been shown that earlier proposed defect models may require further improvement to simulate possible modifications of sample geometrical and thermal properties in defect sites.
\end{abstract}

\section{Introduction}

In military, composites are increasingly used in manufacturing light ballistic protections which withstand well bullets and small arm fragments. Such composites are typically made on the basis of very resistant aramide and polyethane fibers joined with phenolic and polyurethane resins and other elastic mixtures. They are lightweight, non-corrosive and flexible to fit well surface to be protected. Composites can be also applied in conjunction with steel sheets and ceramics, thus increasing their protecting efficiency. Also, damaged composite armour can be easily replaced with new ones without disassembling a total protection system [1, 2, 3].

The defects which can appear in the above-mentioned composites are typically deficiencies in glue layers, as well as stratifications and delaminations occurring if a composite is impacted by 'hard objects'.

Thermal nondestructive testing (NDT) is regarded as a fairly relevant technique for detecting defects in composite materials. Both modeling and data processing aspects of thermal NDT are well explored in the case of Carbon Fiber Reinforced Plastics (CFRP), Glass Fiber Reinforced Plastics (GFRP) and CarbonCarbon (CC) composites [4]. In this paper, the accent is made on some inspection and modeling peculiarities which require further improvement of defect models to simulate possible modifications of sample geometrical and thermal properties in defect sites.

\section{Materials and samples}

Five samples made of aramide layers $(0.6 \mathrm{~mm}$-thick $)$ joined with formaldehyde resin glue (0.04 mm-thick) were inspected (see figure 1 and table 1$)$. Defects were simulated by Teflon inserts and air gaps. All samples were subjected to both front$(\mathrm{F})$ and rear $(\mathrm{R})$-surface tests ( $\tau_{h}$-heat pulse duration; $Q$-heat power density). 
Samples \# 1 \& 3

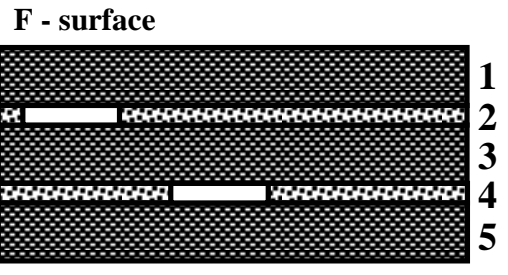

R - surface
Samples \# 2, 4 \& 5

F - surface

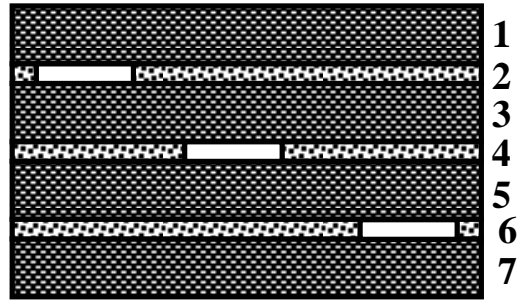

R - surface

Layers 1, 3, 5, 7 - Aramide (0.6 mm)

Layers 1, 3, 5, 7 - Aramide (0.6 mm)

Layers 2, 4, 6 - Formaldehyde resin $(0.04 \mathrm{~mm})$

Layers 2, 4, 6 - Formaldehyde resin $(0.04 \mathrm{~mm})$ Defects - Teflon (Air)

Fig. 1. Sample schemes

The thermal properties of the materials are assumed as follows: aramide conductivity perpendicular to fibers $\lambda_{1}=0.142 \mathrm{~W} /(\mathrm{m} \cdot \mathrm{K})$; conductivity parallel to fibers $\quad \lambda_{\|}=1.69 \mathrm{~W} /(\mathrm{m} \cdot \mathrm{K})$; density $\rho=1330 \mathrm{~kg} / \mathrm{m}^{3}$; heat capacity $C=1047 \mathrm{~J} /(\mathrm{kg} \cdot \mathrm{K})$; diffusivity perpendicular to fibers $\alpha_{\mathrm{L}}=0.1 \cdot 10^{-6} \mathrm{~m}^{2} / \mathrm{s}$; diffusivity parallel to fibers $\lambda_{\|}=1.19 \cdot 10^{-6} \mathrm{~m}^{2} / \mathrm{s}$; formaldehyde resin $\lambda=0.2 \mathrm{~W} /(\mathrm{m} \cdot \mathrm{K}) ; \quad \rho=1200 \mathrm{~kg} / \mathrm{m}^{3} ; C=1850 \mathrm{~J} /(\mathrm{kg} \cdot \mathrm{K}) ; \quad \alpha=2.22 \cdot 10^{-6} \mathrm{~m}^{2} / \mathrm{s} ;$ air (in thin gaps) - $\lambda=0.07 \mathrm{~W} /(\mathrm{m} \cdot \mathrm{K}) ; \rho=1.2 \mathrm{~kg} / \mathrm{m}^{3} ; C=1005 \mathrm{~J} /(\mathrm{kg} \cdot \mathrm{K})$; $\alpha=5.8 \cdot 10^{-5} \mathrm{~m}^{2} / \mathrm{s} ; \quad$ Teflon $\quad-\quad \lambda=0.23 \mathrm{~W} /(\mathrm{m} \cdot \mathrm{K}) ; \quad \rho=2210 \mathrm{~kg} / \mathrm{m}^{3}$; $C=1050 \mathrm{~J} /(\mathrm{kg} \cdot \mathrm{K}) ; \alpha=0.99 \cdot 10^{-7} \mathrm{~m}^{2} / \mathrm{s}$.

\section{Basic models}

The samples analyzed have been modeled by using ThermoCalc- $6 \mathrm{~L}$ software (Innovation, Ltd., Russia) which allows introducing up to six different material layers and up to nine defects of arbitrary shape and thickness within the corresponding 3D thermal NDT problem. Due to the limit in the number of layers, only five-layers samples can be simulated directly, and Model \#1 is equivalent to that in figure 1 on the left (Samples \#1 \& 3). Samples \#2, 4 \& 5 have been simulated by Model \#2 and Model \#3 respectively (figure 2). It is seen that these models are slightly different depending on whether the F- or R-surface is considered. All three models have been verified by using Multilayer-1D software (Innovation, Ltd., Russia) which allows numerical analysis of 1D structures with a unlimited number of layers. On the Fsurface, all models have produced identical results with data divergence under $0.2 \%$, while on the R-surface Model \#3 has revealed accuracy of few percent, i.e. better than Model \#2 due to more correct values of total layer thermal resistances. All models represent a 3D non-adiabatic multi-layer anisotropic heat conduction problem. However, theoretical predictions produced by the models above have not 
been in a good accordance with the experimental results, therefore, a deeper analysis has been done to match theory and the experiment.

The results obtained on samples \#2, 4 \& 5 (Models \# 2 \& 3) are being prepared for other publication. In this paper, the accent is made on analyzing samples \#1 \& 3 (Model \#1).

\section{Model \#1 (F \& R-surface tests)}

\section{Identical to figure 1}

Model \#2 (F-surface test)

F - surface

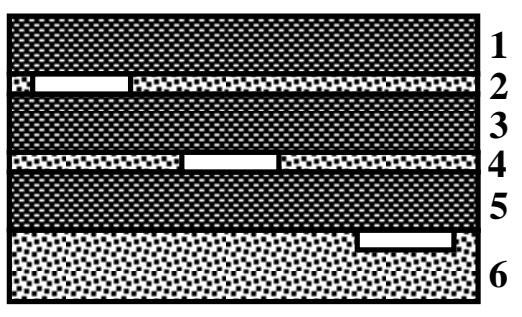

$\mathbf{R}$ - surface

Layers 1, 3, 5 - Aramide (0.6 mm)

Layers 2, 4, 6 - Formaldehyde resin $(0.04 \mathrm{~mm})$

Defects - Teflon (Air)

\section{Model \#3 (R-surface test)}

F - surface

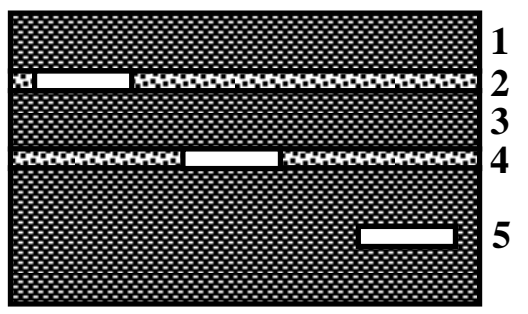

R - surface

Layers 1, 3, 5 - Aramide $(0.6 \mathrm{~mm})$

Layers 2, 4 - Formaldehyde resin $(0.04 \mathrm{~mm})$

Defects - Teflon (Air)

Fig. 2. Sample models

Table 1. Tested composite samples

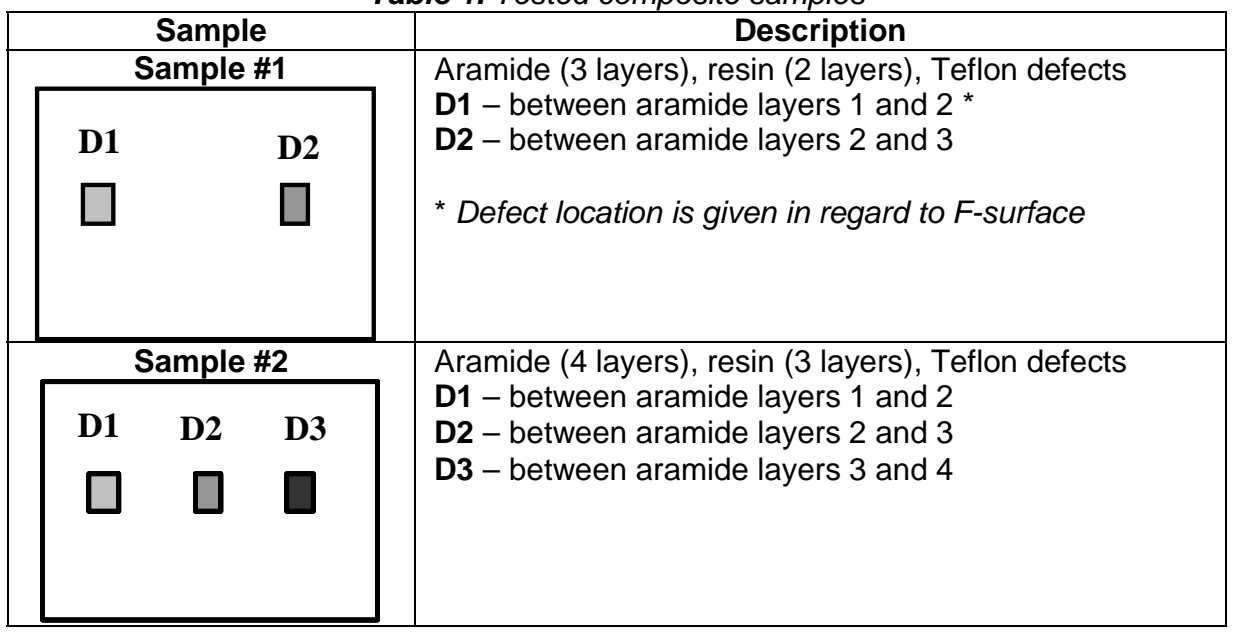


Table 1 continued

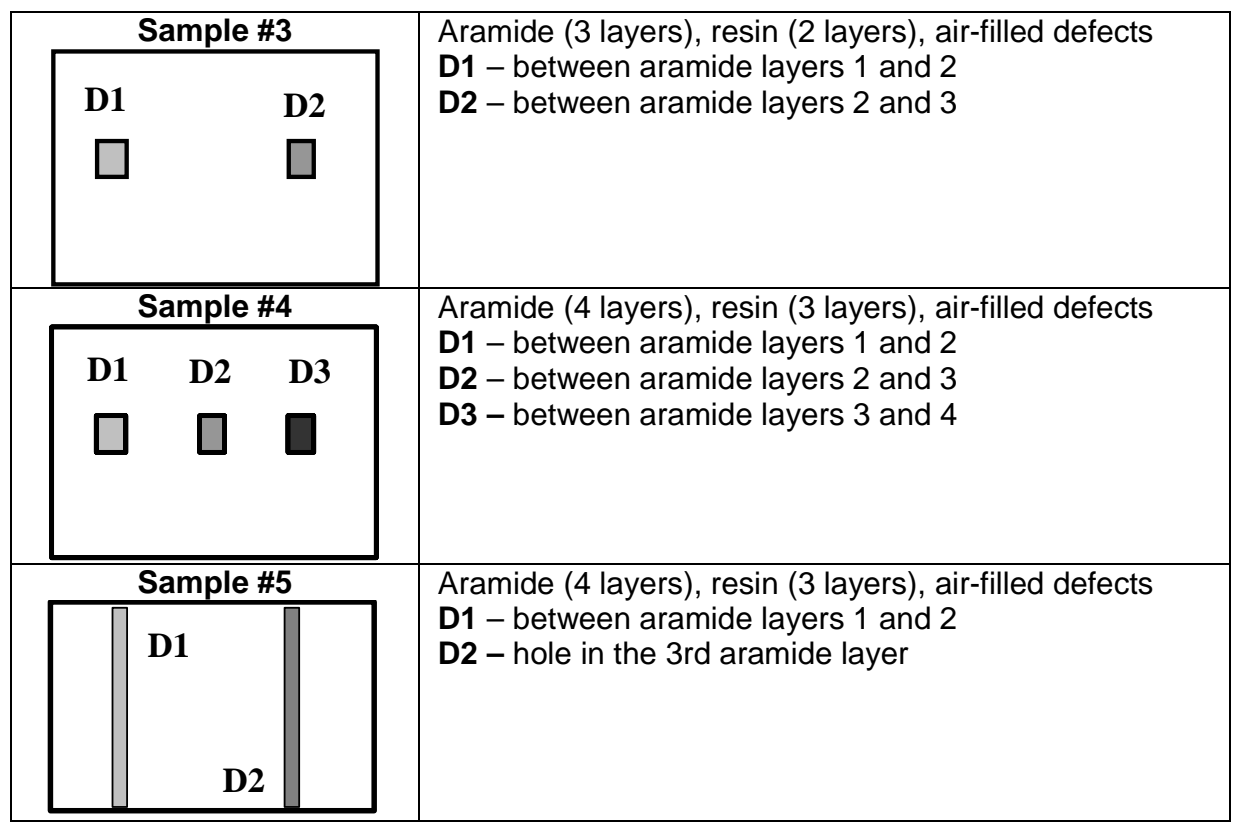

\section{Experimental results}

Samples were optically heated with a set of lamps that allowed sample Fsurface excess temperature to reach $65^{\circ} \mathrm{C}$ for $\tau_{h}=3 \mathrm{~s}$; thus absorbed energy density was estimated to be $Q=3.2 \mathrm{~kW} / \mathrm{m}^{2}$. Temperature was monitored with a Thermovision 900 IR thermographic system. The experimental results obtained on all samples are shown in figure 3 where the left column represents 'optimal' raw images and the right column contains phasegrams obtained by applying the Fourier transformation in time (for the 1st non-zero frequency); this technique is often called Pulse Phase Thermography (PPT). The images were qualitatively evaluated by an operator to produce the results in table 2.
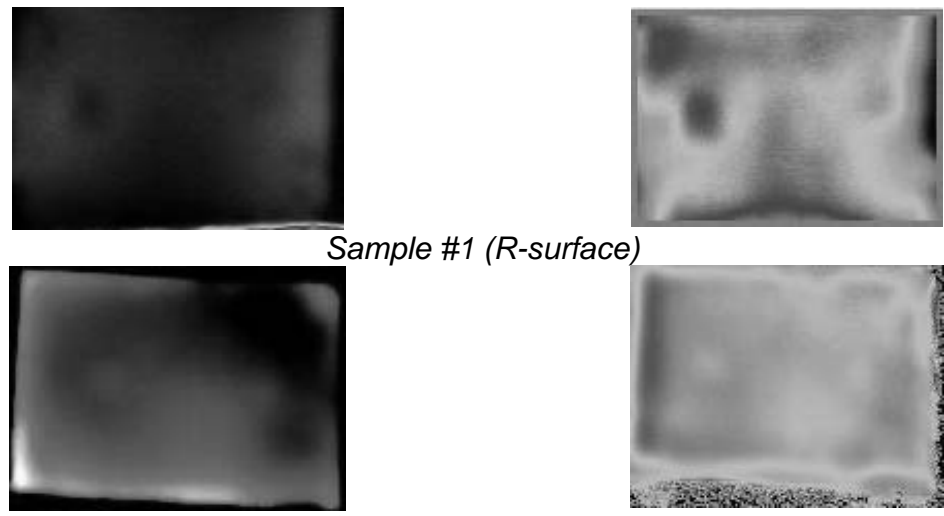

Sample \#1 (R-surface)

Sample \#1 (F-surface)

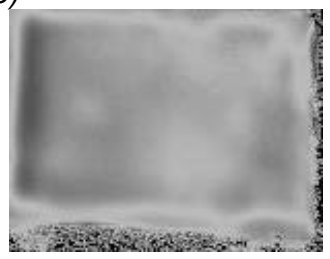

Fig. 3 to continue 
http://dx.doi.org/10.21611/qirt.2006.045

Fig. 3 continued

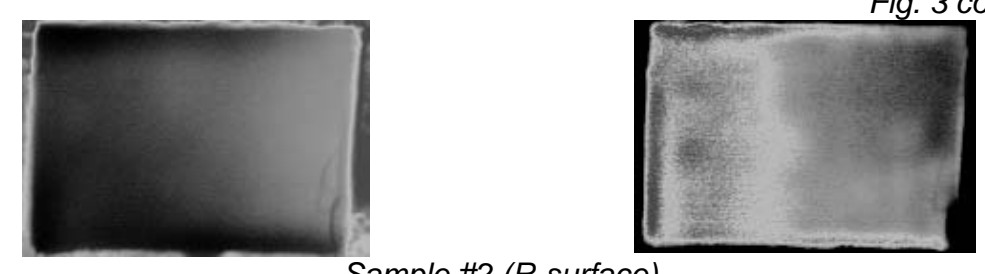

Sample \#2 (R-surface)

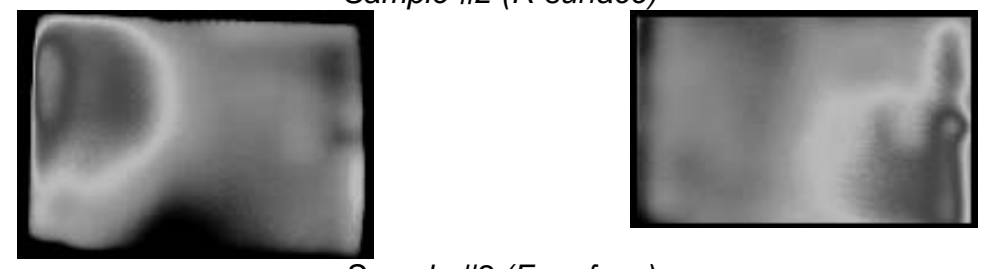

Sample \#2 (F-surface)

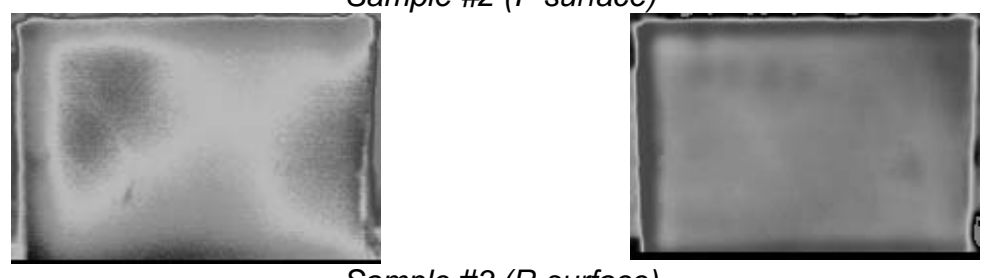

Sample \#3 (R-surface)

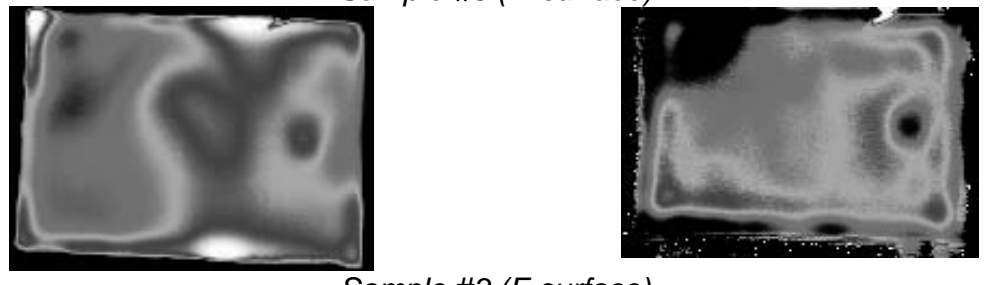

Sample \#3 (F-surface)

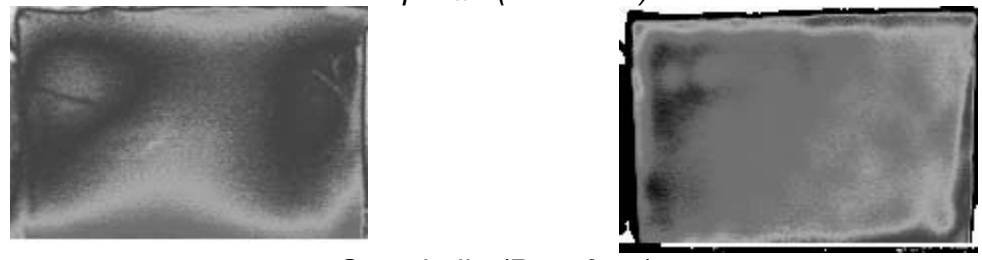

Sample \#4 (R-surface)

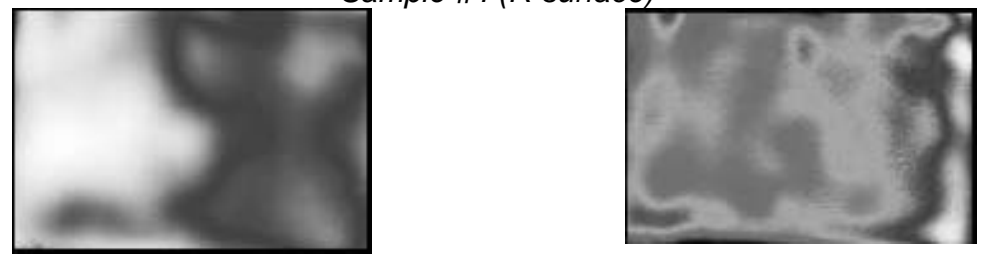

Sample \#4 (F-surface)

Fig. 3 to continue 


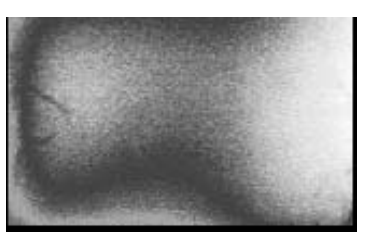

Fig. 3 continued

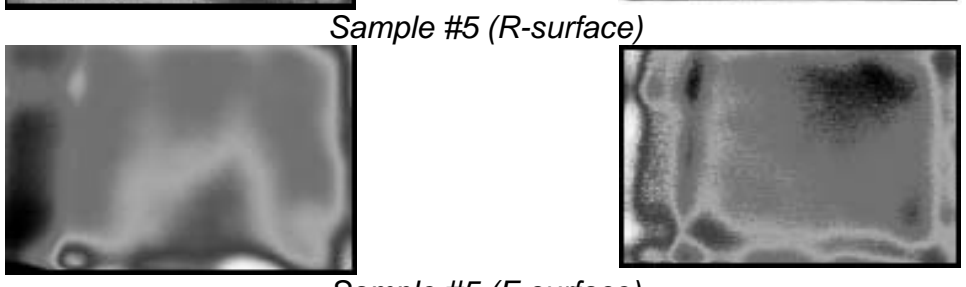

Sample \#5 (F-surface)

Fig. 3. Experimental results for Samples \#1 to \#5

Table 2. Quantitative evaluation of the data in figure 3

\begin{tabular}{|c|c|c|}
\hline \multirow[t]{2}{*}{ Sample } & \multicolumn{2}{|c|}{ Defect signal description } \\
\hline & F-surface & R-surface \\
\hline$\# 1$ & $\begin{array}{c}P^{*} \\
(\mathrm{D} 1, \mathrm{D} 2)\end{array}$ & $\begin{array}{c}\mathbf{N}^{* *} \\
(\mathrm{D} 1, \mathrm{D} 2)^{* \star *}\end{array}$ \\
\hline \#2 & $\begin{array}{c}P \\
\text { (D3) }\end{array}$ & $\begin{array}{c}-{ }^{* \star * \star} \\
(\mathrm{D} 1, \mathrm{D} 3)\end{array}$ \\
\hline \#3 & $\begin{array}{c}P \\
\text { (D2) }\end{array}$ & $(-)^{\star \star \star \star}$ \\
\hline$\# 4$ & $\overline{(-)}$ & $\overline{(-)}$ \\
\hline$\# 5$ & $\begin{array}{c}P \\
\text { (D1) }\end{array}$ & $\dot{-}$ \\
\hline
\end{tabular}

* $\quad \mathbf{P}$ means that the defect produces positive $\Delta T$

** $\mathbf{N}$ means that the defect produces negative $\Delta T$

*** Defect(s) detected by phasegram

**** Defect(s) not detected

The following conclusions can be made.

- The temporal evolution of differential temperature signals, defined as $\Delta T=T_{d}-T_{n d}$ ( $T_{d}$-temperature in a defect area, $T_{n d}$-temperature in a nondefect area), reveals $\Delta T>0$ on F-surface and $\Delta T<0$ on R-surface over both Teflon inserts and air-filled gaps. However, since Teflon is a little more conductive than formaldehyde resin, it may be expected than the sign of $\Delta T$ should be opposite in case of Teflon inserts.

- In some test cases, defect signals have been low and thus some defects have been undetectable on a noisy background, probably, due to close thermal properties of involved materials.

- $\quad$ F-surface test results have seemed to be a little better than R-surface results.

- Best defect detection has been provided by applying the PPT technique. 


\section{Modeling test cases}

First, the test cases described above were simulated by using the ThermoCalc-6L numerical program which proved to be relevant in thermal NDT of thicker composites. However, two immediate difficulties appeared while comparing calculated and experimental data:

- Teflon inserts being in ideal thermal contact with aramide used to produce $\Delta T<0$ on F-surface and $\Delta T>0$ on R-surface, thus contradicting the experimental data.

- $\quad$ Suprisingly, simulated air-filled defects used to produce $\Delta T>0$ on both $\mathrm{F}$ and R-surface.

The first difficulty was overcome by introducing air gaps between Teflon inserts and aramide according to the model discussed in [4]. In such case, the temporal behavior of $\Delta T$ became consistent with experimental observations in figure 3.

The second difficulty was thourighly analyzed by using some other computing algorithms to produce the identical result, i.e. $\Delta T>0$ on R-surface that means better heat conduction through air than through resin. Omitting discussion on how it can be physically possible, for example, due to high air diffusivity, we have noticed that in thin samples two defect models should be considered: 1) an air-filled defect substitutes a piece of a host material as it is assumed in the above-mentioned computer programs, thus not changing the sample thickness $L$ (Defect model \#1 in figure 4a), 2) an air-filled defect increases sample thickness by the defect thickness $d$ (Defect model \#2 in figure $4 b$ ).

Both models have been comparatively studied by using the ThermoCalc-2D software (Innovation, Ltd.). The results are presented in figure 5 and table 3 . The difference between two models is clearly seen; for example, Defect model \#2 leads to negative $\Delta T$ values on R-surface as it was observed in the experiment. It is worth reminding that, in case of Defect model \#2, the sample thickness is increased by $d=0.04 \mathrm{~mm}$ in the defect site.

Defect model\#1

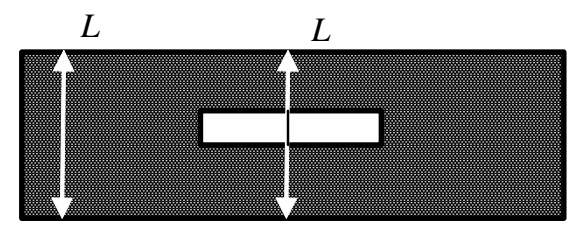

Defect model\#2

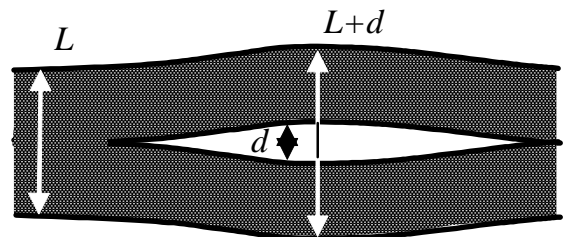

Fig. 4. Modeling defects in composites:

a - Defect model \#1 (a defect substitutes a host material, sample thickness remains the same)

$b$ - Defect model \#2 (a defect increases a sample thickness)

It is expected that in thicker composites both models will lead to similar results on F-surface but in a R-surface test procedure, a defect model should be evaluated carefully. Also, some other fcators may appear when modeling real test cases, 
namely, compression or enlargement of a sample, or modification of host material thermal properties while producing artificial defects, etc.

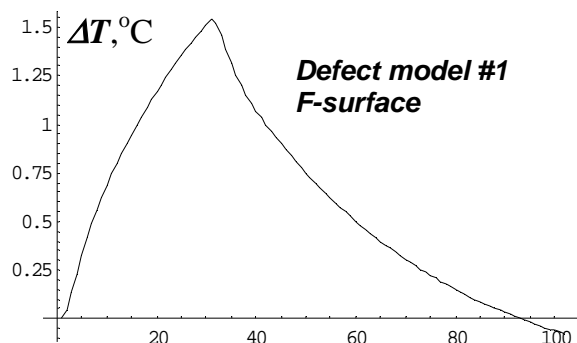

a)

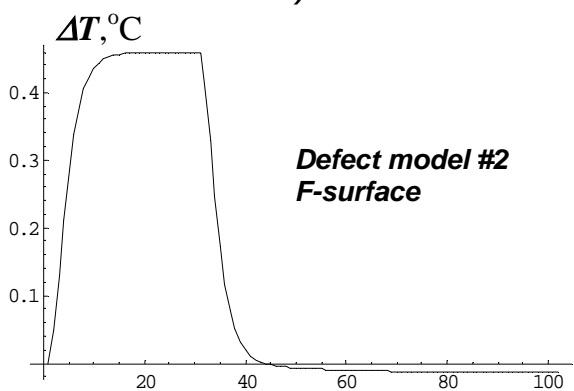

c)

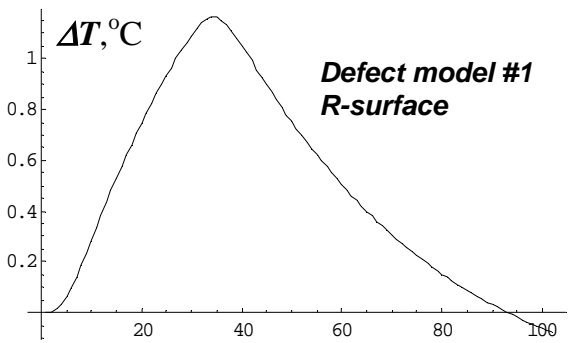

b)

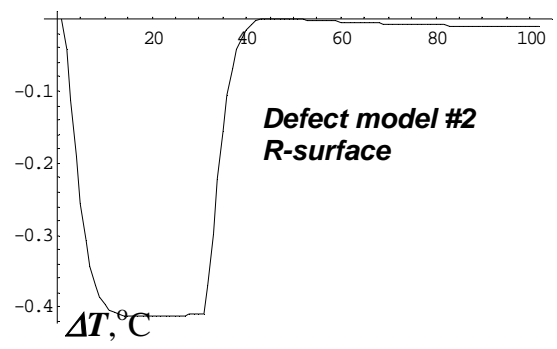

d)

Fig. 5. Modeling defects in aramide composite by figure 4: (sample thickness $0.6+0.6+0.04 \mathrm{~mm}$ in case of Defect model \#1 and $0.6+0.6+0.04+0.04 \mathrm{~mm}$ in case of Defect model \#2, air-filled defect thickness $0.04 \mathrm{~mm}$, heating time $3 \mathrm{~s}$, heat power density $3.2 \mathrm{~kW} / \mathrm{m}^{2}$ ):
a-Defect model \#1, F-surface,
$b$ - Defect model \#1, R-surface,
$c$-Defect model \#2, F-surface,
$d$-Defect model \#2, R-surface

Table 3. Comparing defect models (test case in figure 5)

\begin{tabular}{|c|c|c|c|c|}
\hline \multirow{2}{*}{ Defect model } & \multicolumn{2}{|c|}{ F-surface } & \multicolumn{2}{c|}{ R-surface } \\
\cline { 2 - 5 } & $\Delta T_{m} *,{ }^{\circ} \mathrm{C}$ & $\tau_{m}, \mathrm{~s}$ & $\Delta T_{m},{ }^{\circ} \mathrm{C}$ & $\tau_{m}, \mathrm{~s}$ \\
\hline$\# 1$ & +1.54 & 3.0 & +1.17 & 3.3 \\
\hline$\# 2$ & +0.46 & 2.2 & -0.41 & 1.6 \\
\hline
\end{tabular}

* $\Delta T_{m}$ is the maximum differential temperature signal

** $\tau_{m}$ is the time when $\Delta T_{m}$ appears 


\section{Conclusions}

- $\quad$ Thermal NDT can be applied for evaluating thin polyaramide composites but appearing temperature signals might be low that requires using advanced data processing techniques, such as Pulse Phase Thermography.

- In a one-sided test, defect detectability appeared to be better than in a twosided test, probably, due to close thermal properties of materials involved and higher temperature signals on F-surface.

- In layered polyaramide structures, simulating defects in formaldehyde resin layers with Teflon or air gaps might lead to inconsistent results because of combination of some factors, such as sample compression or enlargement, modification of sample material thermal properties, etc. Modeling thermal NDT in this case requires further improvement of existing numerical and analytical models.

\section{REFERENCES}

[1] W. Świderski, and W. Habaj. Thermography - applications to the testing of bullet protection and ballistic limit V50 for composite armours based on reinforced plastics, Proc. International Conference on Quality Control by Artificial Vision, Vol.1, (May21-23, 2001), pp. 49-53.

[2] W. Świderski. Lock-in thermography to rapid evaluation of destruction area in composite materials used in military applications, SPIE Proc. "ThermosenseXXV', Vol. 5132 (2003), pp. 506-517.

[3] W. Świderski. Infrared inspection of light composite armours with small arms impacts, Męzinarodni Konference Pyrotechniku, Luhacovice, Poland (2002), pp.127-132.

[4] V. Vavilov, D.D. Burleigh, and A. Klimov. Advanced modeling of thermal NDT problems: from buried landmines to defects in composites, Proc. SPIE "Thermosense-XXIV", Vol. 4710 (2002), pp. 507-521. 